\title{
The polio eradication endgame: Why immunization and continued surveillance is critical
}

\author{
Booth $\mathrm{TF}^{1,2 *}$, Grudeski $\mathrm{E}^{1}$, McDermid $\mathrm{A}^{1,2}$, Rotondo $\mathrm{J}^{3}$ \\ ${ }^{1}$ Viral Diseases Division, National Microbiology Laboratory, Public Health Agency of Canada, Winnipeg, MB \\ ${ }^{2}$ Department of Medical Microbiology, University of Manitoba, Winnipeg, MB \\ ${ }^{3}$ Center for Immunization and Respiratory Infectious Diseases, Public Health Agency of Canada, Ottawa, ON
}

*Correspondence: tim.booth@phac-aspc.gc.ca

\begin{abstract}
The poliovirus is very close to being eradicated from the world. To this end, the four main objectives of the World Health Organization's Polio Eradication \& Endgame Strategic Plan 2013-2018 are to: detect and interrupt all poliovirus transmission; strengthen immunization systems and withdraw oral polio vaccine; contain poliovirus and certify interruption of transmission; and plan polio's legacy. There is a need to maintain vigilance for circulating vaccine-derived polioviruses as well as maintaining both epidemiological and laboratory surveillance for polio at this critical point in history. Despite the elimination of indigenous wild poliovirus transmission in Canada, the risk of wild poliovirus importation from endemic countries, and the risk of importation of circulating vaccine strains remains. Due to this ongoing risk, active surveillance of acute flaccid paralysis (AFP) in children less than 15 years of age remains important. At least one stool specimen from all suspect AFP cases should be sent to the National Microbiology Laboratory at the Public Health Agency of Canada for polio isolation and testing to support and verify Canada's polio-free status. An added benefit of this is that it may also help identify other non-polio enteroviruses, such as enterovirus D68.
\end{abstract}

\section{Introduction}

Poliomyelitis is a highly infectious viral disease that can cause swift and irreversible paralysis, particularly in children. Since the introduction of polio vaccines in the 1950s, and the Global Polio Eradication Initiative in 1988, a strategy was developed to eliminate and to eventually eradicate poliovirus infection from the world, through the use of intensive and persistent immunization programs in every country. This vaccination strategy has been highly successful. The World Health Organization (WHO) has stated that, as a result of immunization, there are 10 million people walking today who would otherwise have been paralyzed (1). There have been recent setbacks, however, and in May 2014 the WHO declared the international spread of wild poliovius to be a "Public Health Emergency of International Concern" (2). As recently as September 2015, new cases of polio have been reported in Mali and the Ukraine (3).

The objective of this article is to: summarize the current situation; identify the recent challenges to the elimination strategy; identify new vaccine recommendations from the WHO; and highlight the importance of acute flaccid paralysis (AFP) surveillance around the world, including here in Canada.

\section{Polioviruses and the different vaccines}

Poliovirus has three major strains or serotypes (PV1, PV2 and PV3) and is a member of the C group of enteroviruses in the picornavirus family, a diverse group of positive-stranded RNA viruses, which include many other non-polio enteroviruses (4). It is spread from person to person largely through the fecal-oral route. Approximately $90 \%$ of cases are asymptomatic, $10 \%$ have mild infection (fever, malaise, headache, nausea and vomiting), and less than $1 \%$ of cases develop an acute onset of flaccid paralysis that usually involve the lower limbs. When it involves respiratory muscles, it can be life-threatening (5). 
There are two types of vaccine: oral polio vaccine (OPV) and injectable polio vaccine (IPV). Oral polio vaccine initially contained attenuated versions of all three strains, but PV2 will soon be omitted, due to the eradication of wild PV2. The injectable (inactivated) polio vaccine has drawbacks, including that it is more expensive, more difficult logistically to administer, and is less effective than the Sabin OPV. This is why OPV is still used in much of the world. The concern about OPV, however, is that its use can cause the circulation of vaccine-derived (specifically OPV-derived) polioviruses in populations. These vaccine-derived polioviruses often circulate asymptomatically in populations where oral polio vaccines are still used, and water sanitation is not optimal. These circulating OPV-derived polioviruses invariably revert, though mutation, to a form with increased neurovirulence. Infection by these viruses poses an increased risk of poliomyelitis, especially in persons that are immunocompromised or who have other underlying illnesses.

The resurgence of polio is not a high-profile issue in Canada, where IPV vaccine is used with recommended doses at 2, 4, 6 and 12 to 18 months of age, with a booster at 4 to 6 years of age. This is, however, a global problem and, in light of this, a single lifetime booster dose is now recommended for those at increased risk of exposure to polio (e.g., those travelling to, or planning to work in areas that have wild polio or vaccinederived polio outbreaks) (6).

\section{Polio is re-emerging}

A series of setbacks have occurred in the battle to rid the world of polio, resulting in polio spreading back into areas where it had been previously eliminated. To date, each time this has occurred, the outbreak has been contained through enhanced vaccination, but the battle is not yet over.

The outbreak in Tajikistan in 2010 (7) illustrates the complexity of eradicating the disease. Laboratory genetic analysis identified the causative agent as poliovirus 1 strain. The virus originated in India, and subsequently spread into the neighbouring countries of Kazakhstan, Russia, Turkmenistan and Uzbekistan. This large outbreak of poliomyelitis resulted in 463 laboratory-confirmed cases and 47 polio-compatible cases, and highlights the need for continued polio vaccination, polio campaigns and ongoing surveillance for polio, even in areas where vaccination rates are still high.

Despite these outbreaks due to imported viruses, the number of regions where wild polioviruses are endemic have steadily diminished. In 2012, there were only 223 polio cases identified worldwide, the fewest that have ever been recorded (8), and in 2013 polio was found to be endemic in only three countries-Afghanistan, Nigeria and Pakistan. By early 2014, polio was declared to have been eliminated from Southeast Asia, and in Africa was on the verge of elimination. It was apparent that the risk of outbreaks of paralytic disease arose from circulating vaccine-derived viruses from OPV—and had to be tackled.

In 2014, outbreaks began to occur in regions where polio had formerly been eliminated, including Cameroon, Equatorial Guinea, Ethiopia, Iraq, Israel, Somalia, and Syria (2).

In the Middle East, the outbreaks were caused by a poliovirus 1 strain that had spread from Pakistan. The WHO declared the international spread of wild poliovirus a Public Health Emergency of International Concern after documentation of numerous poliovirus exportations from polio-endemic countries (Pakistan, Afghanistan and Nigeria) to polio-free countries. Enhanced vaccination campaigns and travel requirements were introduced to control the spread of infection in these affected areas. See Table 1 for a summary of the current global polio situation (2). 
Table 1:Revised designations* for infected states from the sixth meeting of the IHR Emergency Committee regarding the international spread of wild poliovirus.

\begin{tabular}{|l|l|}
\hline \multicolumn{1}{|c|}{ Designation } & \multicolumn{1}{c|}{ States } \\
\hline States currently exporting wild poliovirus & Afghanistan, Pakistan \\
\hline $\begin{array}{l}\text { States no longer infected by wild poliovirus, but } \\
\text { remain vulnerable to international spread }\end{array}$ & $\begin{array}{l}\text { Nigeria, Somalia, Cameroon, Equatorial Guinea, } \\
\text { Ethiopia, Iraq, Israel, Syria }\end{array}$ \\
\hline
\end{tabular}

${ }^{\star}$ As of September $24^{\text {th }}, 2015$.

\section{Children with poliovirus infections are still being diagnosed in Canada}

Since 2005, there have been five importations of vaccine-strain poliovirus into Canada that were confirmed by the National Microbiology Laboratory (NML) of the Public Health Agency of Canada (Appendix). Four of these were incidental infections, in that the children were sampled for virological testing due to a nonparalytic illness and poliovirus was cultured from patient specimens. These individuals became infected with vaccine strains during travel to regions where oral vaccines were still in use, either by directly receiving the vaccine, or possibly becoming infected through environmental contamination. One case of vaccineassociated paralytic poliomyelitis was identified which was associated with a child that travelled to China on a vacation and received OPV while there, and presented with flaccid paralysis soon afterwards.

\section{The endgame strategy}

To address the setbacks with polio, the WHO developed the Polio Eradication \& Endgame Strategic Plan 2013-2018 that contains four main objectives: detect and interrupt all poliovirus transmission; strengthen immunization systems and withdraw oral polio vaccine; contain poliovirus and certify interruption of transmission; and plan polio's legacy (1). We will focus on the vaccine recommendations and the need for detection of all poliovirus transmission.

\section{Phasing out oral vaccine}

In early 2014, the WHO recommended all countries use at least one dose of IPV (8). The reasoning for this is to prevent outbreaks of circulating OPV-derived polioviruses, which can result in cases of OPV-related poliomyelitis. Although IPV gives good humoral immunity and protection from poliomyelitis disease, it gives much less intestinal immunity. Thus, about $90 \%$ of children vaccinated with IPV are still prone to shedding the virus after being given OPV. Thus, the use of IPV alone would probably not be enough to eradicate circulating OPV-derived viruses. It has been shown that giving children a dose of IPV first, followed by booster of OPV, greatly reduced the shedding of OPV-derived virus in stool: the results were much better than giving a dose of OPV first and a second booster of OPV (9).

The WHO also recommended that OPV vaccine no longer include PV2 (10). This was based on data that showed wild polio PV2 is probably extinct (11) and wild PV3 has not been detected since 2012 (12). Removal of PV3 from OPV may follow the discontinuation of the PV2 component.

\section{Acute flaccid paralysis (AFP) surveillance}

Surveillance for poliovirus relies on two things: the detection, reporting and stool testing of AFP in children or paralysis in any person who is suspected of being infected with poliomyelitis; and a laboratory that can positively identify poliovirus and distinguish wild polio from vaccine-derived disease. Nationwide AFP surveillance is the WHO gold standard for detecting cases of poliomyelitis (13).

As a member of the Global Poliovirus Laboratory Network, the NML is responsible for the virological testing of stool specimens from cases with a clinical suspicion of poliomyelitis. The WHO stipulates that stool samples from at least $80 \%$ of AFP cases in children less than 15 years of age should undergo virological 
stool testing for poliovirus. Unfortunately, over the last decade or so, only about $30 \%$ of these cases have undergone laboratory testing, and very few of these tests were carried out by an accredited laboratory. This is the weak link in our current surveillance system.

To have a strong AFP surveillance system in Canada it is important to report cases of AFP in children under 15 years and submit stool specimens to the NML which is the only WHO-accredited laboratory for poliovirus testing in Canada. Details on how to submit specimens for testing at the NML are included below. This is important to do even when another plausible cause for the illness is found. For the diagnosis of poliovirus infection, virus culture from stool (in a special cell line that is highly sensitive to poliovirus) is still the most sensitive standard recommended by the WHO to identify polio. In addition, molecular testing, including reverse-transcription polymerase chain reaction testing to detect the viral genome, and genetic sequencing, is carried out to type and identify the origin of any polioviruses that are detected.

\section{What clinicians need to know}

Acute flaccid paralysis (AFP) is defined as the acute onset of focal weakness or paralysis characterised as flaccid (reduced tone) without other obvious cause (e.g., trauma) in children less than 15 years old (14). AFP is nationally notifiable. Any attending physician who diagnoses AFP in children less than 15 years should report cases to the Canadian Pediatric Surveillance Program (CPSP) and to local public health in provinces and territories where AFP is notifiable by law (15). The CPSP has recently updated its AFP protocol and questionnaire (14). It is a best practice to arrange a 60-day follow up.

At least one stool sample needs to be collected within 14 days of the onset of paralysis and if more than one specimen is taken they should be 24-48 hours apart. No special medium is required; stool can be collected in any sterile leak-proof container. There should be at least $1 \mathrm{~g}$ and ideally $5 \mathrm{~g}$ to $10 \mathrm{~g}$ of stool for each sample. A cold chain is needed; samples need to be stored frozen at $\leq-20^{\circ} \mathrm{C}$ and then shipped frozen on dry ice by those who are known to be compliant with Transportation of Dangerous Goods Regulations. An easier alternative (that does not require special transportation) is to send the stool sample surrounded by an ice pack that has been kept overnight in a $-80^{\circ} \mathrm{C}$ freezer.

Stool samples are typically sent through the provincial public health laboratory, but samples can also be shipped directly to the NML using an NML shipping account; NML will cover the charges. To arrange this, contact the corresponding author by e-mail or telephone (204-789-6067). A requisition can be downloaded. It is most useful to include relevant clinical findings, travel and vaccination history, as well any relevant lab results that are available. Once samples arrive at the Enterovirus and Enteric Virus Section of the NML, multiple tests are done, including viral isolation in cell culture; typing of poliovirus isolates; screening for potential vaccine-derived polioviruses; and sequencing to confirm the presence of a wild-type, a Sabin-like or a vaccine-derived poliovirus strains. The total turnaround time is 28 days.

\section{An added benefit of AFP surveillance}

Reporting and systematic stool testing of AFP cases in Canada can do more than document our polio-free status. Non-polio flaccid paralysis is frequently associated with non-polio enteroviruses (16), and these viruses can also cause encephalitis, meningitis and encephalomyelitis (17-19). For example, enterovirus A71 (EV-A71) has been causing periodic outbreaks of hand, foot and mouth disease in children, especially in Asia $(20,21)$. There are non-polio enteroviruses and novel syndromes that may be associated with emerging enteroviruses. Most non-polio enteroviruses affect children disproportionately, and some are potentially fatal.

An outbreak of enterovirus D68 (EV-D68) occurred in Canada and the United States in association with mostly mild respiratory illness in children (22-27). It has been suggested that acute flaccid myelitis (an unexplained neurological illness involving limb weakness in children) could be associated with EV-D68, since an increased incidence of these cases observed in the U.S. coincided with the increased detection of EV-D68 in 2014 (28). 
These outbreaks underline the need to carry out continued and enhanced laboratory-based and epidemiological surveillance for AFP.

\section{Discussion}

The global battle to eradicate the poliovirus is now at a critical point. The risk remains of the introduction of wild poliovirus and OPV-derived poliovirus in Canada and elsewhere from countries where it still is endemic. Due to this ongoing risk, vaccine coverage of the entire population and active surveillance of AFP in children less than 15 years of age continues to be critical.

Three things are essential to the eradication of polio: first, maintaining a high level of immunity in the population; second, ongoing vigilance, reporting and stool testing of all cases of AFP; and, third, accurate laboratory diagnostics to detect importations of poliovirus into Canada.

In addition to the need for better stool testing and reporting of AFP cases, this article identifies a gap in national polio surveillance activities. Currently, non-paralytic poliovirus infections in individuals who have not been recently vaccinated with OPV do not meet the national poliomyelitis case definition (29). As such, the list of poliovirus infections (Appendix) of which the Public Health Agency of Canada is aware of at this time may be incomplete. A review of provincial and territorial case definitions indicates that non-paralytic poliovirus infections are only notifiable in Ontario at this time (30). Given the emphasis on identifying and reporting all poliovirus infections, a revision to the national case definition to include such cases may be indicated.

Although many people believe the end of polio is now in sight, it may still take years of hard effort and vigilant surveillance before both wild PV1 and circulating OPV-derived polioviruses can finally be declared eradicated. Once eradication is achieved, there will be huge benefits in that the costs of continued global polio vaccination can eventually be saved and the infrastructure that was developed for polio eradication can then be devoted to addressing other health issues.

\section{Conclusion}

The WHO's new vaccine recommendations and acute flaccid paralysis surveillance are both critical to eradicating polio. Canada's ongoing laboratory- and epidemiological-based polio surveillance is important for maintaining polio-free certification at a critical stage in world polio eradication. However, this can only happen with ongoing clinical vigilance for AFP that includes appropriate reporting and testing protocols.

\section{Acknowledgements}

The authors wish to thank Susan Squires, Mary Louise Graham and Rukshanda Ahmad for their input on the clinical aspects of the paper and to Rhiannon Huzarewich, Lily MacDonald, Michelle Gusdal, Russel Mandes, Helen Bangura and Shelley Deeksfor their excellent technical assistance.

\section{Conflict of interest}

None

\section{References}

(1) World Health Organization (WHO). Polio Eradication \& Endgame Strategic Plan 2013-2018. Geneva: WHO; 2013. http://www.polioeradication.org/Portals/O/Document/Resources/StrategyWork/PEESP_EN_US.pdf

(2) World Health Organization (WHO). Statement on the $6^{\text {th }}$ IHR Emergency Committee meeting regarding the international spread of wild poliovirus. Geneva: WHO; 2015 August 17. http://www.who.int/mediacentre/news/statements/2014/polio-20140505/en

(3) Roberts L. Polio resurfaces in Mali and Ukraine. Science 8 September 2015. DOI: 10.1126/science.aad1723. http://news.sciencemag.org/health/2015/09/polio-resurfaces-mali-and-ukraine 
(4) van Regenmortel MHV, Fauquet CM, Bishop DHL, Carsten EB, Estes MK, Lemon SM, et al., editors. Virus Taxonomy: Seventh Report of the International Committee on Taxonomy of Viruses. San Diego, CA: Academic Press; 2000.

(5) Heymann DL, editor. Control of Communicable Diseases Manual. 20th ed. Washington, DC: American Public Health Association; 2014

(6) Public Health Agency of Canada. Canadian Immunization Guide. Part 4-Active Vaccines: Poliomyelitis Vaccine [updated 2015 Feb 25].

http://www.phac-aspc.gc.ca/publicat/cig-gci/p04-poli-eng.php

(7) Yakovenko ML, Gmyl AP, Ivanova OE, Eremeeva TP, Ivanov AP, Prostova MA, et al. The 2010 outbreak of poliomyelitis in Tajikistan: Epidemiology and lessons learnt. Euro Surveill. 2014 Feb 20;19(7):20706.

(8) Centers for Disease Control and Prevention (CDC). Progress toward interruption of wild poliovirus transmissionworldwide, January 2011-March 2012. MMWR 2012 May 18;61(19):353-7.

(9) Jafari H, Deshpande JM, Sutter RW, Bahl S, Verma H, Ahmad M, et al. Efficacy of inactivated poliovirus vaccine in India Science 22 August 2014: 345 (6199), 922-925. [DOI:10.1126/science.1255006]

(10) World Health Organization (WHO). Polio vaccines: WHO position paper, January 2014. Wkly Epidemiol Rec. 2014 Feb 28;89(9):73-92.

http://www.who.int/wer/2014/wer8909.pdf?ua=1

(11) World Health Organization (WHO). Transmission of wild poliovirus type 2-apparent global interruption. Wkly Epidemiol Rec. 2001 Mar 30;76 (13): 95-7.

(12) Kew OM, Cochi SL, Jafari HS, Wassilak SGF, Mast EE, Diop OM, et al. Possible eradication of wild poliovirus type 3 -worldwide, 2012. MMWR. 2014 Nov 14;63(45):1031-3.

(13) Wassilak SG, Oberste MS, Tangermann RH, Diop OM, Jafari HS, Armstrong GL. Progress toward global interruption of wild poliovirus transmission, 2010-2013, and tackling the challenges to complete eradication. J Infect Dis. 2014 Nov; 210 Suppl 1:S5-15. doi:10.1093/infdis/jiu456.

(14) Canadian Paediatric Surveillance Program. Acute flaccid paralysis. http://www.cpsp.cps.ca/surveillance/studyetude/acute-flaccid-paralysis.

(15) Public Health Agency of Canada. Poliomyletis (Polio): Surveillance http://www.phac-aspc.gc.ca/im/vpdmev/poliomyelitis/surveillance-eng.php

(16) Rao CD, Yergolkar P, Shankarappa KS. Antigenic diversity of enteroviruses associated with nonpolio acute flaccid paralysis. India, 2007-2009. Emerg Infect Dis. 2012

Nov;18(11). http://wwwnc.cdc.gov/eid/article/18/11/11-1457_article

(17) Rhoades RE, Tabor-Godwin JM, Tsueng G, Feuer R. Enterovirus infections of the central nervous system review. Virology. 2011 Mar 15;411(2):288-305. doi: 10.1016/j.virol.2010.12.014. Epub 2011 Jan 20.

(18) Lum LC, Wong KT, Lam SK, Chua KB, Goh AY, Lim WL, et al. Fatal enterovirus 71 encephalomyelitis. J Pediatr. 1998 Dec;133(6):795-8.

(19) Michos AG, Syriopoulou VP, Hadjichristodoulou C, Daikos GL, Lagona E, Douridas P, Mostrou G, Theodoridou M. Aseptic meningitis in children: Analysis of 506 cases. PLoS One. 2007 Aug 1; 2(7):e674.

(20) Wu W-H, Kuo T-C, Lin Y-T, Huang S-W, Liu H-F, Wang J, Chen Y-M A. Molecular epidemiology of enterovirus 71 infection in the central region of Taiwan from 2002 to 2012. PLoS One. 2013 Dec 31;8(12):e83711. doi: 10.1371/journal.pone.0083711.

(21) China on alert as virus spreads. BBC News. 2008 May 3. http://news.bbc.co.uk/2/hi/asia-pacific/7381741.stm

(22) Midgley CM, Jackson MA, Selvarangan R, Turabelidze G, Obringer E, Johnson D, et al. Severe respiratory illness associated with enterovirus D68-Missouri and Illinois, 2014. MMWR. 2014 Sep 12;63(36):798-9. http://www.cdc.gov/mmwr/preview/mmwrhtml/mm6336a4.htm

(23) Human enterovirus D68-North America (20): Update. ProMED. 2014 Dec 15. Archive No: 20141215.3035008. http://www.promedmail.org/direct.php?id=3035008

(24) Pastula DM, Aliabadi N, Haynes AK, Messacar K, Schreiner T, Maloney J, et al. Acute neurologic illness of unknown etiology in children-Colorado, August-September 2014. MMWR. 2014 Oct 10;63(40):901-2. http://www.cdc.gov/mmwr/preview/mmwrhtml/mm6340a5.htm

(25) Fonseca K, Kellner JD, Talbot J, Simmonds K, Anselmo M, Kuhn S, et al. Human enterovirus 68-Canada: (Alberta). Archive No. 20140916.2775534. 2014 Sep 16. http://www.promedmail.org/direct.php?id=2775534

(26) BC Centre for Disease Control. British Columbia Influenza Surveillance Bulletin. Influenza Season 2014-15, Number 9, Weeks 48-49, November 23 to December 6, 2014. 2014 Dec 11. http://www.bccdc.ca/NR/rdonlyres/35012AE9-2753-4A27-9DD587B3A11A40DC/0/InfluBulletin_Number09_Weeks4849_201415.pdf.

(27) Centers for Disease Control and Prevention. Enterovirus D68 in the United States, 2014. http://www.cdc.gov/non-polio-enterovirus/outbreaks/EV-D68-outbreaks.html

(28) Roos R., Center for Infectious Disease Research and Policy (CIDRAP). Link between polio-like illness and EVD68 looks likely. CIDRAP News, 2014 Dec 2. http://www.cidrap.umn.edu/news-perspective/2014/12/cdc-linkbetween-polio-illness-and-ev-d68-looks-likely 
(29) Public Health Agency of Canada. Case definitions for communicable diseases under national surveillance. Poliomyelitis. CCDR. 35S2. http://www.phac-aspc.gc.ca/publicat/ccdr-rmtc/09vol35/35s2/Polio-eng.php

(30) National Collaborating Centre for Infectious Diseases. Notifiable Disease Database. Accessed on July 24, 2014. http://nddb.ca/en/diseaseinfo/list

(31) Desai S, Diener T, Tan BJ-K, Lowry NJ, Talukdar C, Chrusch WM. An unusual case of vaccine-associated paralytic poliomyelitis. Can J Infect Dis Med Microbiol. 2014;25(4):227-8.

(32) Bartlett M, Murti M, Gubbay J, Khan A, Bontavics E, Simpson M, Booth T, Dolman S, Yaffe B, Chong-King E, Desai S, Finkelstein M. $9^{\text {th }}$ Canadian Immunization Conference. Abstracts. Public health response to detection of poliovirus in Toronto. Can J Infect Dis Med Microbiol. 2010;21(4):14. 
Appendix: Identified poliovirus infections in Canada, 2004 to $2015^{1}$

\begin{tabular}{|c|c|c|c|c|c|}
\hline Year & $\begin{array}{c}\text { Virology } \\
\text { test result }\end{array}$ & Symptoms & Source & $\begin{array}{c}\text { Recent travel } \\
\text { history }\end{array}$ & $\begin{array}{c}\text { Outcomel } \\
\text { diagnosis }\end{array}$ \\
\hline $2004^{2}$ & Polio Sabin 1 & Diarrhoea & Stool culture & India & Incidental infection \\
\hline $2005^{2}$ & Polio Sabin 3 & $\begin{array}{c}\text { Urinary tract } \\
\text { infection }\end{array}$ & Stool culture & Unknown & Incidental infection \\
\hline 2009 & Polio Sabin 3 & $\begin{array}{c}\text { Acute flaccid } \\
\text { paralysis }\end{array}$ & Stool culture & China & $\begin{array}{c}\text { Probable case of } \\
\text { vaccine-associated } \\
\text { paralytic } \\
\text { poliomyelitis (31) }\end{array}$ \\
\hline 2009 & Polio Sabin 3 & $\begin{array}{c}\text { Fever and } \\
\text { cough }\end{array}$ & $\begin{array}{c}\text { Nasopharyngeal } \\
\text { culture }\end{array}$ & India & $\begin{array}{c}\text { Incidental infection } \\
\text { (32) }\end{array}$ \\
\hline $2012^{2}$ & Polio Sabin 2 & Diarrhoea & Stool culture & Philippines & Incidental infection \\
\hline
\end{tabular}

